Теорія Ймовір. та Матем. Статист. Вип. 76, 2007
Theor. Probability and Math. Statist.

No. 76, 2008, Pages 23-31

S 0094-9000(08)00728-X

Article electronically published on July 10, 2008

\title{
A LOCATION INVARIANT MOMENT-TYPE ESTIMATOR. I
}

\author{
UDC 519.21
}

CHENG-XIU LING, ZUOXIANG PENG, AND SARALEES NADARAJAH

\begin{abstract}
The moment's estimator (Dekkers et al., 1989) has been used in extreme value theory to estimate the tail index, but it is not location invariant. The location invariant Hill-type estimator (Fraga Alves, 2001) is only suitable to estimate positive indices. In this paper, a new moment-type estimator is studied, which is location invariant. This new estimator is based on the original moment-type estimator, but is made location invariant by a random shift. Its weak consistency and strong consistency are derived, in a semiparametric setup.
\end{abstract}

\section{INTRODUCTION}

Suppose $X_{1}, X_{2}, \ldots, X_{n}$ are i.i.d. random variables with common distribution function (d.f.) $F(x)$ and let $X_{1, n} \leq X_{2, n} \leq \cdots \leq X_{n, n}$ be the associated order statistics. If there exist some numbers $a_{n}>0, b_{n} \in \mathbb{R}$ and some non-degenerate distribution $G(x)$ such that

$$
\mathrm{P}\left(X_{n, n} \leq a_{n} x+b_{n}\right)=F^{n}\left(a_{n} x+b_{n}\right) \stackrel{d}{\rightarrow} G(x) \quad \text { as } n \rightarrow \infty,
$$

then $G(x)$ must be equivalent to

$$
G_{\gamma}(x)= \begin{cases}\exp \left\{-(1+\gamma x)^{-1 / \gamma}\right\}, & 1+\gamma x>0, \gamma \neq 0 \\ \exp \{-\exp (-x)\}, & x \in \mathbb{R}, \gamma=0\end{cases}
$$

If $F$ satisfies (1.1) we say $F(x)$ belongs to the domain of attraction of an extreme value d.f. $G_{\gamma}$, denoted by $F \in D\left(G_{\gamma}\right)$ and $\gamma$ is referred to as the extreme value index (EVI). In the last two decades many estimators of the extreme value index $\gamma \in \mathbb{R}$ have been proposed that use upper order statistics; see, for example, Hill [16], Pickands 20], Dekkers et al. [6], Drees [7, 8, and Drees and Kaufmann [9]. For maximum likelihood estimators of $\gamma$, see Hall [15], Smith [24, 25], and Smith and Weissman [26]. For $\gamma>0$, Hill [16] proposed the estimator given by

$$
\hat{\gamma}_{n}^{H}(k)=\frac{1}{k} \sum_{i=0}^{k-1} \log X_{n-i, n}-\log X_{n-k, n}
$$

for $k=1, \ldots, n-1$. For $\gamma \in \mathbb{R}$, Dekkers et al. [6] proposed the moment-type estimators

$$
\hat{\gamma}_{n}^{M}=M_{n}^{(1)}+1-\frac{1}{2}\left\{1-\frac{\left(M_{n}^{(1)}\right)^{2}}{M_{n}^{(2)}}\right\}^{-1},
$$

2000 Mathematics Subject Classification. Primary 60F99.

Key words and phrases. Extreme value index, location invariant property, moment estimation, strong and weak consistencies, order statistics, regular varying functions. 
where

$$
M_{n}^{(j)}=\frac{1}{k} \sum_{i=0}^{k-1}\left(\log \frac{X_{n-i, n}}{X_{n-k, n}}\right)^{j}
$$

for $j=1,2$. Pan [17] discussed the asymptotic expansion of the distribution of the Hill and moment estimators. Cheng and de Haan [1] and Cuntz et al. 3] obtained penultimate forms of the Hill estimator.

The above estimators are scale invariant but not location invariant. Indeed there are mathematical as well as practical reasons to require location invariance properties. Since an affine transformation of the r.v.'s $X_{i}$ merely leads to a change of the normalizing constants $a_{n}$ and $b_{n}$, it influences neither the extreme value index nor the accuracy of the approximation (1.1). Moreover, in practice, the observations (e.g., sea levels, temperatures) depend on an arbitrarily chosen zero-point, which equally should not affect the estimator. In fact, the prominent Pickands [20] estimator given by

$$
\hat{\gamma}_{n}^{P}=\frac{1}{\log 2} \log \frac{X_{n-k+1, n}-X_{n-2 k+1, n}}{X_{n-2 k+1, n}-X_{n-4 k+1, n}}
$$

is both scale and location invariant, where $k=k(n)$ is an intermediate integer sequence, i.e., $k=k(n) \rightarrow \infty$ and $k / n \rightarrow 0$. Qi and Cheng [21] and Peng [19] discussed the asymptotic behavior of various Pickands-type estimators. Segers [23] proposed a general Pickands estimator given by

$$
\hat{\gamma}_{n, k}(c, v)=\frac{1}{\log v} \log \left(\frac{X_{n-[c k], n}-X_{n-k, n}}{X_{n-[c v k], n}-X_{n-[v k], n}}\right)
$$

and proved its consistency, asymptotic normality and discussed an optimal choice of $c$ and $v$ in the sense of minimum square error (MSE). Drees 8 proposed a general class of estimators which have the scale invariant property. For $\gamma>0$, Fraga Alves [1] established a location invariant Hill estimator given by

$$
\hat{\gamma}_{n}^{H}\left(k_{0}, k\right)=\frac{1}{k_{0}} \sum_{i=0}^{k_{0}-1} \log \left(\frac{X_{n-i, n}-X_{n-k, n}}{X_{n-k_{0}, n}-X_{n-k, n}}\right),
$$

where $k \rightarrow \infty, k_{0} \rightarrow \infty, k / n \rightarrow 0, k_{0} / k \rightarrow 0$, and discussed its weak consistency, asymptotic expansion and the optimal choice of the sample fraction $k_{0}$.

Although both (1.4) and (1.5) are location invariant, (1.4) has poor efficiency and it is difficult to decide on the optimal sample fraction $k$. Also (1.5) is only valid for $\gamma>0$. In this paper, we propose a general estimator for $\gamma \in \mathbb{R}$ based on the invariant Hill estimator and the moment-type estimator. It is given by

$$
\hat{\gamma}_{n}^{M}\left(k_{0}, k\right)=M_{n}^{(1)}\left(k_{0}, k\right)+1-\frac{1}{2}\left\{1-\frac{\left(M_{n}^{(1)}\left(k_{0}, k\right)\right)^{2}}{M_{n}^{(2)}\left(k_{0}, k\right)}\right\}^{-1},
$$

where

$$
M_{n}^{(j)}\left(k_{0}, k\right)=\frac{1}{k_{0}} \sum_{i=0}^{k_{0}-1}\left(\log \frac{X_{n-i, n}-X_{n-k, n}}{X_{n-k_{0}, n}-X_{n-k, n}}\right)^{j}
$$

for $j=1,2$ and $k=k(n), k_{0}=k_{0}(n)$ are integer sequences that satisfy $0<k \leq n$, $0<k_{0} \leq k$. We derive the weak and strong consistencies of this new estimator (Section 2). 


\section{WEAK AND STRONG CONSISTENCY}

In what follows we assume that $F \in D\left(G_{\gamma}\right), \gamma \in \mathbb{R}$, which is equivalent to supposing that $U:=(1 /(1-F))^{\leftarrow}$ is a general regularly varying function with index $\gamma$ (denoted by $\left.U \in G R V_{\gamma}\right)$; i.e., there exists a measurable function $a(t)>0$ such that as $t \rightarrow \infty$,

$$
\frac{U(t x)-U(t)}{a(t)} \rightarrow \frac{x^{\gamma}-1}{\gamma} \quad(=\log x \text { for } \gamma=0)
$$

holds for all $x>0$ (de Haan [4]). In fact, the auxiliary function $a(t)$ is a regularly varying function at infinity with index $\gamma$, denoted by $a(t) \in R V_{\gamma}$.

Let $Y_{1}, \ldots, Y_{n}$ be independent random variables (r.v.'s) with common d.f.

$$
F_{Y}(y)=1-1 / y, \quad y \geq 1,
$$

and let $Y_{1, n} \leq Y_{2, n} \leq \cdots \leq Y_{n, n}$ be the associated order statistics. Then $X_{(i, n)} \stackrel{d}{=} U\left(Y_{i, n}\right)$ for $i=1, \ldots, n$. Notice also that the following relations are true:

$$
\left\{\frac{Y_{n-i, n}}{Y_{n-k_{0}, n}}\right\}_{i=0}^{k_{0}-1} \stackrel{d}{=}\left\{Y_{k_{0}-i, k_{0}}\right\}_{i=0}^{k_{0}-1}
$$

and

$$
\log Y_{i, n} \stackrel{d}{=} E_{i, n}
$$

for $i=1, \ldots, n$, where $E_{i, n}$ is the $i$ th order statistic associated with a random sample $E_{1}, \ldots, E_{n}$ from the standard exponential distribution. Notice further that for any intermediate sequence $k=k(n)$, as $n \rightarrow \infty,(k / n) Y_{n-k, n} \rightarrow 1$ in probability, and that for any integer sequence $k(n)$ satisfying $k(n) / n \rightarrow 0$ and $k(n) /(\log n)^{\delta} \rightarrow \infty$ for some $\delta>0$, as $n \rightarrow \infty,(k / n) Y_{n-k, n} \rightarrow 1$ almost surely.

Now we present the main results concerning the proposed estimator's consistency.

Theorem 2.1. If $U \in G R V_{\gamma}, x^{*}(F)>0$ (the right endpoint of $F$ ), $k(n) / n \rightarrow 0$, $k_{0} / k \rightarrow 0, k(n) \rightarrow \infty$ and $k_{0}(n) \rightarrow \infty$ as $n \rightarrow \infty$, then

$$
\lim _{n \rightarrow \infty} \hat{\gamma}_{n}^{M}\left(k_{0}, k\right)=\gamma
$$

in probability.

Theorem 2.2. If $U \in G R V_{\gamma}, x^{*}(F)>0, k(n) / n \rightarrow 0, k_{0} / k \rightarrow 0, k /(\log n)^{\delta_{1}} \rightarrow \infty$ and $k_{0} /(\log k)^{\delta_{2}} \rightarrow \infty$ for some $\delta_{1}, \delta_{2}>0$, then

$$
\lim _{n \rightarrow \infty} \hat{\gamma}_{n}^{M}\left(k_{0}, k\right)=\gamma
$$

almost surely.

For the proof we need some lemmas.

Lemma 2.1. Let $U(t) \in G R V_{\gamma}$ and $x_{*}=\inf \{x: F(x)>0\}$. There exists a function $f\left(t_{2}, t_{1}\right)$ such that as $t_{1} \rightarrow \infty$ and $t_{2} / t_{1} \rightarrow \infty$

$$
\frac{1}{f\left(t_{2}, t_{1}\right)} \log \frac{U\left(t_{2} s\right)-U\left(t_{1}\right)}{U\left(t_{2}\right)-U\left(t_{1}\right)} \longrightarrow \begin{cases}\log s, & \gamma \geq 0 \\ \left(s^{\gamma}-1\right) / \gamma, & \gamma<0\end{cases}
$$

holds locally uniformly for all $s>0$. Moreover for every $\varepsilon>0$, there exist $N_{1}, N_{2}$ such that $t_{1}>N_{1}, t_{1} s \geq N_{1}, t_{2} / t_{1}>N_{2}$,

$$
(1-\varepsilon) \frac{1-s^{-\varepsilon}}{\varepsilon}-\varepsilon<\frac{1}{f\left(t_{2}, t_{1}\right)} \log \frac{U\left(t_{2} s\right)-U\left(t_{1}\right)}{U\left(t_{2}\right)-U\left(t_{1}\right)}<(1+\varepsilon) \frac{s^{\varepsilon}-1}{\varepsilon}+\varepsilon
$$


provided that $\gamma \geq 0$, and

$$
1-(1+\varepsilon) s^{\gamma+\varepsilon}<\frac{1}{f\left(t_{2}, t_{1}\right)} \log \frac{U\left(t_{2} s\right)-U\left(t_{1}\right)}{U\left(t_{2}\right)-U\left(t_{1}\right)}<1-(1-\varepsilon) s^{\gamma-\varepsilon}
$$

provided that $\gamma<0$.

Remark 2.1. In Lemma 2.1 we can take the function $f\left(t_{2}, t_{1}\right)$ to satisfy

$$
f\left(t_{2}, t_{1}\right) \sim \frac{a\left(t_{2}\right)}{U\left(t_{2}\right)-U\left(t_{1}\right)} \sim \begin{cases}\gamma, & \gamma>0, \\ \log ^{-1}\left(t_{2} / t_{1}\right), & \gamma=0, \\ (-\gamma)\left(t_{2} / t_{1}\right)^{\gamma}, & \gamma<0,\end{cases}
$$

where $a(t)$ is the auxiliary function of $U(t)$. In particular, $f\left(t_{2}, t_{1}\right)>0$ and

$$
f\left(t_{2}, t_{1}\right) \rightarrow \max (\gamma, 0)
$$

as $t_{1} \rightarrow \infty$ and $t_{2} / t_{1} \rightarrow \infty$.

Proof. We prove (2.5) for the three cases $\gamma>0, \gamma=0$ and $\gamma<0$.

(i) Consider $\gamma>0$. For $0<s \leq 1$,

$$
U\left(t_{2} s\right)-U\left(t_{2}\right) \leq 0 \text {. }
$$

As $t_{1}, t_{2} / t_{1} \rightarrow \infty$ and for all $0<\varepsilon<1$, there exists $N_{1}$ such that $t_{1}>N_{1}, t_{1} s \geq N_{1}$, $t_{1} / t_{2}<\varepsilon$ and

$$
U\left(t_{2}\right)-U\left(t_{2} \varepsilon\right)<U\left(t_{2}\right)-U\left(t_{1}\right)<U\left(t_{2}\right) .
$$

Combining (2.8) and (2.9),

$$
\frac{U\left(t_{2} s\right)-U\left(t_{2}\right)}{U\left(t_{2}\right)-U\left(t_{2} \varepsilon\right)}<\frac{U\left(t_{2} s\right)-U\left(t_{2}\right)}{U\left(t_{2}\right)-U\left(t_{1}\right)}<\frac{U\left(t_{2} s\right)-U\left(t_{2}\right)}{U\left(t_{2}\right)} .
$$

Letting $t_{2} \rightarrow \infty$ in (2.10),

$$
\frac{s^{\gamma}-1}{1-\varepsilon^{\gamma}} \leq \liminf \frac{U\left(t_{2} s\right)-U\left(t_{2}\right)}{U\left(t_{2}\right)-U\left(t_{1}\right)} \leq \lim \sup \frac{U\left(t_{2} s\right)-U\left(t_{2}\right)}{U\left(t_{2}\right)-U\left(t_{1}\right)} \leq s^{\gamma}-1 .
$$

Letting $\varepsilon \downarrow 0$ in (2.11),

$$
\frac{U\left(t_{2} s\right)-U\left(t_{2}\right)}{U\left(t_{2}\right)-U\left(t_{1}\right)} \rightarrow s^{\gamma}-1
$$

Thus,

$$
\log \frac{U\left(t_{2} s\right)-U\left(t_{1}\right)}{U\left(t_{2}\right)-U\left(t_{1}\right)}=\log \left(\frac{U\left(t_{2} s\right)-U\left(t_{2}\right)}{U\left(t_{2}\right)-U\left(t_{1}\right)}+1\right) \rightarrow \log \left(s^{\gamma}-1+1\right)=\gamma \log s .
$$

The proof is similar for $s>1$.

(ii) Consider $\gamma=0$. Note that

$$
\frac{a\left(t_{2}\right)}{U\left(t_{2}\right)-U\left(t_{1}\right)}=\frac{1}{\left(U\left(t_{2}\right)-U\left(t_{1}\right)\right) / a\left(t_{2}\right)} \sim \log ^{-1} \frac{t_{2}}{t_{1}} \rightarrow 0
$$

since $\log (1+x) \sim x$ as $x \rightarrow 0$, and

$$
\frac{U\left(t_{2} s\right)-U\left(t_{2}\right)}{a\left(t_{2}\right)} \rightarrow \log s
$$

as $t_{2}, t_{2} / t_{1} \rightarrow \infty$. The result follows by rewriting

$$
\log \frac{U\left(t_{2} s\right)-U\left(t_{1}\right)}{U\left(t_{2}\right)-U\left(t_{1}\right)}=\log \left(\frac{U\left(t_{2} s\right)-U\left(t_{2}\right)}{a\left(t_{2}\right)} \frac{a\left(t_{2}\right)}{U\left(t_{2}\right)-U\left(t_{1}\right)}+1\right) .
$$

(iii) Consider $\gamma<0$. Given $N>1$, there exists $N_{1}$ such that $t_{1}>N_{1}, t_{2} / t_{1}>N$ and

$$
U\left(t_{1} N\right)-U\left(t_{1}\right) \leq U\left(t_{2}\right)-U\left(t_{1}\right) \leq U(\infty)-U\left(t_{1}\right) .
$$


If $0<s \leq 1$, then we get from (2.8) that

$$
\frac{U\left(t_{2} s\right)-U\left(t_{2}\right)}{U\left(t_{1} N\right)-U\left(t_{1}\right)} \leq \frac{U\left(t_{2} s\right)-U\left(t_{2}\right)}{U\left(t_{2}\right)-U\left(t_{1}\right)} \leq \frac{U\left(t_{2} s\right)-U\left(t_{2}\right)}{U(\infty)-U\left(t_{1}\right)}
$$

which can be rewritten as

$$
\begin{aligned}
-\frac{1}{\gamma} \frac{\left(V\left(t_{2} s\right)-V\left(t_{2}\right)\right) / V\left(t_{2}\right)}{\left(V\left(t_{1} N\right)-V\left(t_{1}\right)\right) / V\left(t_{1}\right)} & \leq \frac{1}{-\gamma V\left(t_{2}\right) / V\left(t_{1}\right)} \frac{U\left(t_{2} s\right)-U\left(t_{2}\right)}{U\left(t_{2}\right)-U\left(t_{1}\right)} \\
& \leq \frac{V\left(t_{2} s\right)-V\left(t_{2}\right)}{\gamma V\left(t_{2}\right)} .
\end{aligned}
$$

Let $V(t)=U(\infty)-U(t)$ so that $V(t) \in R V_{\gamma}$. Letting $t_{1} \rightarrow \infty$ and $t_{2} \rightarrow \infty$ in (2.13) we obtain that

$$
\begin{aligned}
-\frac{1}{\gamma} \frac{s^{\gamma}-1}{N^{\gamma}-1} & \leq \liminf \frac{1}{-\gamma V\left(t_{2}\right) / V\left(t_{1}\right)} \frac{U\left(t_{2} s\right)-U\left(t_{2}\right)}{U\left(t_{2}\right)-U\left(t_{1}\right)} \\
& \leq \limsup \frac{1}{-\gamma V\left(t_{2}\right) / V\left(t_{1}\right)} \frac{U\left(t_{2} s\right)-U\left(t_{2}\right)}{U\left(t_{2}\right)-U\left(t_{1}\right)} \leq \frac{s^{\gamma}-1}{\gamma} .
\end{aligned}
$$

Letting $N \rightarrow \infty$ in (2.14),

$$
\frac{a\left(t_{2}\right)}{U\left(t_{2}\right)-U\left(t_{1}\right)}=\frac{a\left(t_{2}\right)}{V\left(t_{1}\right)-V\left(t_{2}\right)} \sim-\gamma \frac{V\left(t_{2}\right)}{V\left(t_{1}\right)} \rightarrow 0
$$

as $t_{2} / t_{1} \rightarrow \infty$, which proves the result. The proof is similar for $s>1$.

The other results of the lemma follow from Geluk and de Haan [12, page 27.

Lemma 2.2. Let $0<k=k(n) \leq n$ and $k(n) \rightarrow \infty$ as $n \rightarrow \infty$.

(i) If $F(x)=x^{\alpha}, 0<x<1$, for some $\alpha>0$, then

$$
\lim _{n \rightarrow \infty} \frac{1}{k(n)} \sum_{i=1}^{k(n)} \frac{X_{i, n}}{X_{k(n)+1, n}}=\frac{\alpha}{\alpha+1}
$$

in probability.

(ii) If $F(x)=1-x^{-\alpha}, x>1$, for some $\alpha>1$, then

$$
\lim _{n \rightarrow \infty} \frac{1}{k(n)} \sum_{i=1}^{k(n)} \frac{X_{n-i-1, n}}{X_{n-k(n), n}}=\frac{\alpha}{\alpha-1}
$$

in probability.

The proof follows from Lemma 2.4 in Dekkers et al. [6].

Lemma 2.3. Let $0<k=k(n) \leq n$ and $k(n) /(\log n)^{\delta} \rightarrow \infty$ as $n \rightarrow \infty$ for some $\delta>0$.

(i) If $F(x)=x^{\alpha}, 0<x<1$, for some $\alpha>0$, then

$$
\lim _{n \rightarrow \infty} \frac{1}{k(n)} \sum_{i=1}^{k(n)} \frac{X_{i, n}}{X_{k(n)+1, n}}=\frac{\alpha}{\alpha+1}
$$

almost surely.

(ii) If $F(x)=1-x^{-\alpha}, x>1$, for some $\alpha>2(1+\delta) / \delta$, then

$$
\lim _{n \rightarrow \infty} \frac{1}{k(n)} \sum_{i=1}^{k(n)} \frac{X_{n-i-1, n}}{X_{n-k(n), n}}=\frac{\alpha}{\alpha-1}
$$

almost surely.

The proof follows from Lemma 2.3 in Dekkers et al. 6 . 
Proof of Theorem 2.1. We prove (2.3) for the three cases $\gamma>0, \gamma=0$ and $\gamma<0$.

(i) Consider $\gamma>0$. One can write

$$
\begin{aligned}
\hat{\gamma}_{n, j}\left(k_{0}, k\right) & \stackrel{d}{=} \frac{1}{k_{0}} \sum_{i=0}^{k_{0}-1}\left[\log \frac{U\left(Y_{n-i, n}\right)-U\left(Y_{n-k, n}\right)}{U\left(Y_{n-k_{0}, n}\right)-U\left(Y_{n-k, n}\right)}\right]^{j} \\
& =\frac{1}{k_{0}} \sum_{i=0}^{k_{0}-1}\left[\log \frac{\left(Y_{n-i, n} / Y_{n-k, n}\right)^{\gamma}-1}{\left(Y_{n-k_{0}, n} / Y_{n-k, n}\right)^{\gamma}-1}\left(1+o_{p}(1)\right)\right]^{j} \\
& =\frac{1}{k_{0}} \sum_{i=0}^{k_{0}-1}\left[\log Y_{k_{0}-i, k_{0}}^{\gamma} \frac{1-Y_{k-i, k}^{-\gamma}}{1-Y_{k-k_{0}, k}^{-\gamma}}\left(1+o_{p}(1)\right)\right]^{j} \\
& =\frac{1}{k_{0}} \sum_{i=0}^{k_{0}-1}\left[\gamma E_{i}\left(1+o_{p}(1)\right)\right]^{j}
\end{aligned}
$$

which implies that

$$
\hat{\gamma}_{n, 1}\left(k_{0}, k\right) \stackrel{\mathrm{P}}{\rightarrow} \gamma
$$

and

$$
\hat{\gamma}_{n, 2}\left(k_{0}, k\right) \stackrel{\mathrm{P}}{\rightarrow} 2 \gamma^{2}
$$

and thus (2.3) is proved.

(ii) Consider $\gamma=0$. Since $k=k(n), k_{0}=k_{0}(k)$ are intermediate integer sequences and $U(t) \in G R V_{\gamma}$,

$$
\begin{aligned}
\log \frac{U\left(Y_{n-i, n}\right)-U\left(Y_{n-k, n}\right)}{U\left(Y_{n-k_{0}, n}\right)-U\left(Y_{n-k, n}\right)} & =\log \left(1+\frac{U\left(Y_{n-i, n}\right)-U\left(Y_{n-k_{0}, n}\right)}{U\left(Y_{n-k_{0}, n}\right)-U\left(Y_{n-k, n}\right)}\right) \\
& =\frac{\log \left(Y_{n-i, n} / Y_{n-k_{0}, n}\right)}{\log \left(Y_{n-k_{0}, n} / Y_{n-k, n}\right)}\left(1+o_{p}(1)\right) \\
& =\frac{\log Y_{k_{0}-i, k_{0}}}{\log Y_{k-k_{0}, k}}\left(1+o_{p}(1)\right)
\end{aligned}
$$

and

$$
\begin{aligned}
\hat{\gamma}_{n, j}\left(k_{0}, k\right) & =\frac{1}{k_{0}} \sum_{i=0}^{k_{0}-1}\left[\log \frac{U\left(Y_{n-i, n}\right)-U\left(Y_{n-k, n}\right)}{U\left(Y_{n-k_{0}, n}\right)-U\left(Y_{n-k, n}\right)}\right]^{j} \\
& =\frac{1}{\left(\log Y_{k-k_{0}, k}\right)^{j}} \frac{1}{k_{0}} \sum_{i=0}^{k_{0}-1}\left(\log Y_{k_{0}-i, k_{0}}\right)^{j}\left(1+o_{p}(1)\right) .
\end{aligned}
$$

Thus,

$$
\log Y_{k-k_{0}, k} \hat{\gamma}_{n, 1}\left(k_{0}, k\right) \stackrel{\mathrm{P}}{\rightarrow} 1
$$

and

$$
\log ^{2} Y_{k-k_{0}, k} \hat{\gamma}_{n, 2}\left(k_{0}, k\right) \stackrel{\mathrm{P}}{\rightarrow} 2
$$

The result in (2.3) follows by noting that $\log Y_{k-k_{0}, k} \stackrel{\mathrm{P}}{\rightarrow} \infty$. 
(iii) Consider $\gamma<0$. Since $k=k(n), k_{0}=k_{0}(k)$ are intermediate integer sequences and $U(t) \in G R V_{\gamma}$,

$$
\begin{aligned}
\log \frac{U\left(Y_{n-i, n}\right)-U\left(Y_{n-k, n}\right)}{U\left(Y_{n-k_{0}, n}\right)-U\left(Y_{n-k, n}\right)} & =\log \left(1+\frac{U\left(Y_{n-i, n}\right)-U\left(Y_{n-k_{0}, n}\right)}{U\left(Y_{n-k_{0}, n}\right)-U\left(Y_{n-k, n}\right)}\right) \\
& =\frac{\left(Y_{n-i, n} / Y_{n-k_{0}, n}\right)^{\gamma}-1}{1-\left(Y_{n-k_{0}, n} / Y_{n-k, n}\right)^{-\gamma}}\left(1+o_{p}(1)\right) \\
& =\frac{Y_{k_{0}-i, k_{0}}^{\gamma}-1}{1-Y_{k-k_{0}, k}^{-\gamma}}\left(1+o_{p}(1)\right)
\end{aligned}
$$

and so

$$
-\left(1-Y_{k-k_{0}, k}^{-\gamma}\right) \hat{\gamma}_{n, 1}\left(k_{0}, k\right) \stackrel{\mathrm{P}}{\rightarrow} \frac{-\gamma}{1-\gamma}
$$

and

$$
\left(1-Y_{k-k_{0}, k}^{-\gamma}\right)^{2} \hat{\gamma}_{n, 2}\left(k_{0}, k\right) \stackrel{\mathrm{P}}{\rightarrow} \frac{2 \gamma^{2}}{(1-\gamma)(1-2 \gamma)} .
$$

The result in (2.3) follows by noting that $1-Y_{k-k_{0}, k}^{-\gamma} \stackrel{\mathrm{P}}{\rightarrow}-\infty$.

Proof of Theorem 2.2. We prove (2.4) for the two cases $\gamma \geq 0$ and $\gamma<0$ by establishing the limiting behavior of $\hat{\gamma}_{n, j}\left(k_{0}, k\right)$.

(i) Consider $\gamma \geq 0$. Since (2.6) holds, given $\varepsilon>0$, one has by Lemma 2.1 that

$$
\begin{aligned}
& \frac{\hat{\gamma}_{n, j}\left(k_{0}, k\right)}{\left[f\left(Y_{n-k_{0}, n}, Y_{n-k, n}\right)\right]^{j}} \\
& \quad=\frac{1}{k_{0}} \sum_{i=0}^{k_{0}-1}\left\{\frac{1}{f\left(Y_{n-k_{0}, n}, Y_{n-k, n}\right)} \log \frac{U\left(\frac{Y_{n-i, n}}{Y_{n-k_{0}, n}} Y_{n-k_{0}, n}\right)-U\left(Y_{n-k, n}\right)}{U\left(Y_{n-k_{0}, n}\right)-U\left(Y_{n-k, n}\right)}\right\}^{j} \\
& \quad \stackrel{\frac{Y_{n-i, n}^{\varepsilon}}{\text { a.s. }_{n}^{\varepsilon}}-1}{k_{0}} \sum_{i=0}^{k_{0}-1}\left\{\varepsilon+(1+\varepsilon) \frac{Y^{\varepsilon}, n}{\varepsilon}\right.
\end{aligned}
$$

almost surely for all sufficiently large $n$ and for $j=1,2$. First consider $j=1$. Since $Y_{n-i, n}^{\varepsilon}$ is the $(n-i)$ th order statistic from the d.f. $1-1 / x^{1 / \varepsilon}(x>1)$, we can apply Lemma 2.3 for $\varepsilon<\min \left\{\delta_{1} /\left(2\left(1+\delta_{1}\right)\right), \delta_{2} /\left(2\left(1+\delta_{2}\right)\right)\right\}$ and find

$$
\limsup _{n \rightarrow \infty} \frac{\hat{\gamma}_{n, 1}\left(k_{0}, k\right)}{f\left(Y_{n-k_{0}, n}, Y_{n-k, n}\right)} \leq \varepsilon+(1+\varepsilon) \frac{\left\{\frac{\varepsilon^{-1}}{\varepsilon^{-1}-1}-1\right\}}{\varepsilon}
$$

almost surely. This, together with a similar lower inequality, gives

$$
\lim _{n \rightarrow \infty} \frac{\hat{\gamma}_{n, 1}\left(k_{0}, k\right)}{f\left(Y_{n-k_{0}, n}, Y_{n-k, n}\right)}=1
$$

almost surely. Next, because $k Y_{n-k, n} / n \rightarrow 1$ almost surely and $k_{0} Y_{n-k_{0}, n} / n \rightarrow 1$ almost surely, it follows from the property of $f\left(t_{2}, t_{1}\right)$ that

$$
\frac{1}{f\left(n / k_{0}, n / k\right)} f\left(\frac{Y_{n-k_{0}, n}}{n / k_{0}} \frac{n}{k_{0}}, \frac{Y_{n-k, n}}{n / k} \frac{n}{k}\right)=1
$$

almost surely. The case $j=2$ is similar. Thus, for $j=1,2$,

$$
\lim _{n \rightarrow \infty} \frac{\hat{\gamma}_{n, j}\left(k_{0}, k\right)}{\left[f\left(Y_{n-k_{0}, n}, Y_{n-k, n}\right)\right]^{j}}=j !
$$

almost surely. 
(ii) Consider $\gamma<0$. Given $\varepsilon>0$ one finds as in part (i), now using (2.7) in Lemma 2.1, that

$$
\begin{aligned}
& \frac{\hat{\gamma}_{n, j}\left(k_{0}, k\right)}{\left[f\left(Y_{n-k_{0}, n}, Y_{n-k, n}\right)\right]^{j}} \\
& \quad=\frac{1}{k_{0}} \sum_{i=0}^{k_{0}-1}\left\{\frac{1}{f\left(Y_{n-k_{0}, n}, Y_{n-k, n}\right)} \log \frac{U\left(\frac{Y_{n-i, n}}{Y_{n-k_{0}, n}} Y_{n-k_{0}, n}\right)-U\left(Y_{n-k, n}\right)}{U\left(Y_{n-k_{0}, n}\right)-U\left(Y_{n-k, n}\right)}\right\}^{j} \\
& \quad \stackrel{\text { a.s. }}{\quad} \frac{1}{k_{0}} \sum_{i=0}^{k_{0}-1}\left\{1-(1-\varepsilon) \frac{Y_{n-i, n}^{\gamma-\varepsilon}}{Y_{n-k_{0}, n}^{\gamma-\varepsilon}}\right\}^{j}
\end{aligned}
$$

almost surely for all sufficiently large $n$ and for $j=1,2$. Since $Y_{n-i, n}^{\gamma-\varepsilon}$ is the $(i+1)$ st order statistic from the d.f. $x^{1 /(-\gamma+\varepsilon)}(0<x<1)$, one can apply Lemma 2.3 and find that

$$
\limsup _{n \rightarrow \infty} \frac{\hat{\gamma}_{n, 1}\left(k_{0}, k\right)}{\left[f\left(Y_{n-k_{0}, n}, Y_{n-k, n}\right)\right]} \leq 1-(1-\varepsilon) \frac{(\varepsilon-\gamma)^{-1}}{(\varepsilon-\gamma)^{-1}+1}
$$

almost surely. This, together with a similar lower inequality, gives

$$
\lim _{n \rightarrow \infty} \frac{\hat{\gamma}_{n, 1}\left(k_{0}, k\right)}{\left[f\left(Y_{n-k_{0}, n}, Y_{n-k, n}\right)\right]} \rightarrow \frac{-\gamma}{1-\gamma}
$$

almost surely. Following arguments similar to those in part (i), one can now show that

$$
\lim _{n \rightarrow \infty} \frac{\hat{\gamma}_{n, j}\left(k_{0}, k\right)}{\left[f\left(Y_{n-k_{0}, n}, Y_{n-k, n}\right)\right]^{j}}= \begin{cases}-\gamma /(1-\gamma), & j=1, \\ 2 \gamma^{2} /\{(1-\gamma)(1-2 \gamma)\}, & j=2\end{cases}
$$

almost surely.

Acknowledgement. The authors would like to thank the referee and the managing editor for carefully reading the paper and for their great help in improving the paper.

\section{BIBLIOGRAPHY}

1. S. Cheng and L. de Haan, Penultimate approximation for Hill's estimator, Scand. J. Statist. 28 (2001), 569-575. MR.1858418 (2002h:62156)

2. S. Cheng and J. Pan, Asymptotic expansions of estimators for the tail index with applications, Scand. J. Statist. 25 (1998), 717-728. MR1666796 (2000d:62079)

3. A. Cuntz, E. Haeusler, and J. Segers, Edgeworth expansions for the distribution function of the Hill estimator, Discussion paper from Tilburg University, Center for Economic Research (2003), no. 8.

4. L. de Haan, Slow variation and characterization of domains of attraction, Statistical Extremes and Applications, Vimeiro, 1983, Reidel, Dordrecht, 1984, pp. 31-48. MR784812 (87b:60036)

5. L. de Haan and U. Stadtmüller, Generalized regular variation of second order, J. Aust. Math. Soc., A 61 (1996), 381-395. MR1420345 (97g:26001)

6. A. L. M. Dekkers, J. H. J. Einmahl, and L. de Haan, A moment estimator for the index of an extreme-value distribution, Ann. Stat. 17 (1989), 1833-1855. MR.1026315 (91i:62033)

7. H. Drees Refined Pickands estimators of the extreme value index, Ann. Statist. 23 (1995), 2059-2080. MR.1389865 (97d:62069)

8. H. Drees, A general class of estimators of the extreme value index, J. Statist. Plann. Inference 66 (1998), 95-112. MR.1616999 (99c:62085)

9. H. Drees and E. Kaufmann, Selecting the optimal sample fraction in univariate extreme value index, Stochastic Process. Appl. 75 (1998), 149-172. MR1632189(99h:62034)

10. Z. Fan, Estimation problems for distributions with heavy tails, J. Statist. Plann. Inference 123 (2004), 13-40. MR2058119 (2005c:62059)

11. M. I. Fraga Alves, A location invariant Hill-type estimator, Extremes 4 (2001), 199-217. MR.1907061 (2003d:62129)

12. J. L. Geluk and L. de Haan, Regular variation, extensions, and Tauberian theorems, CWI Tract 40 (1987). MR906871(89a:26002) 
13. M. I. Gomes and M. J. Martins, Generalizations of the Hill estimator-asymptotic versus finite sample behavior, J. Statist. Plann. Inference 93 (2001), 161-180. MR.1822394

14. M. I. Gomes and O. Oliveira, Censoring estimators of a positive tail index, Statist. Probab. Lett. 65 (2003), 147-159. MR.2018025 (2005d:62155)

15. P. Hall, On estimating the endpoint of a distribution, Ann. Statist. 10 (1982), 556-568. MR653530 (83f:62043)

16. B. M. Hill, A simple general approach to inference about the tail of a distribution, Ann. Statist. 3 (1975), 1163-1174. MR0378204 (51:14373)

17. J. Pan, Some results on estimation of the tail index of a distribution, Chinese Ann. Math. Ser. B 19 (1998), 239-248. MR 1655939 (2000a:62037)

18. Z. Peng, Extension of Pickands' estimator, Math. Sinica 40 (1997), 759-762. MR1612631 (99c:62111)

19. L. Peng, Asymptotically unbiased estimators for the extreme value index, Statist. Probab. Lett. 38 (1998), 107-115. MR1627906 (99e:62056)

20. J. Pickands, Statistical inference using extreme order statistics, Ann. Statist. 3 (1975), 119-131. MR0423667 (54:11642)

21. Y. Qi and S. Cheng, Convergence of Pickands-type estimators, Chinese Sci. Bull. 37 (1992), 1409-1413.

22. J. Segers, Abelian and Tauberian theorems for the bias of the Hill estimator, Scand. J. Statist. 29 (2001), 461-483. MR.1925570 (2003k:62159)

23. J. Segers, Generalized Pickands estimators for the extreme value index, J. Statist. Plann. Inference 128 (2005), 381-396. MR2102765

24. R. L. Smith, Maximum likelihood estimation in a class of nonregular cases, Biometrika 72 (1985), 67-90. MR790201 (86k:62053)

25. R. L. Smith, Estimation of tails of probability distribution, Ann. Statist. 15 (1987), 1174-1207. MR.902252 (88j:62096)

26. R. L. Smith and I. Weissman, Maximum likelihood estimation of the lower tail of probability distribution, J. Roy. Statist. Soc. Ser. B 47 (1985), 285-298. MR816094 (87b:62035)

27. Z. Tsourti and I. Panaretos, Extreme Value Index Estimators and Smoothing Alternatives: Review and Simulation Comparison, Technical Report, 2001.

28. X. Wang and S. Cheng, General regular variation of nth order and the second order Edgeworth expansion of the extreme value distribution. I, Acta Math. Sin., Engl. Ser. 21 (2005), no. 5, 1121-1130. MR2176324 (2006i:60063)

29. S. Yun, On a general Pickands estimator of extreme value index, J. Statist. Plann. Inference 102 (2002), 389-409. MR1896495 (2003e:62050)

Department of Mathematics, Southwest Normal University, Chongqing 400715, P. R. China

E-mail address: pzx@swu.edu.cn

Department of Mathematics, Southwest Normal University, Chongqing 400715, P. R. China

Department of Statistics, University of Nebraska-Lincoln, Lincoln, Nebraska 68583, USA

E-mail address: snadaraj@unlserve.unl.edu

Received 29/NOV/2005

Originally published in English 